\title{
Excessive Daytime Sleepiness among Hypertensive US-Born Blacks and Foreign-Born Blacks: Analysis of the CAATCH Data
}

\author{
N. Williams, ${ }^{1}$ O. Abo Al Haija, ${ }^{1}$ A. Workneh, ${ }^{1}$ D. Sarpong, ${ }^{2}$ E. Keku, ${ }^{3}$ G. Ogedegbe, \\ S. I. McFarlane, ${ }^{5}$ and G. Jean-Louis ${ }^{1,6}$ \\ ${ }^{1}$ Department of Medicine, Brooklyn Health Disparities Center, SUNY Downstate Medical Center, NY 11203-2098, USA \\ ${ }^{2}$ Jackson State University Medical Systems, Jackson Heart Study, Jackson, MS 39213, USA \\ ${ }^{3}$ Department of Public Health and Preventive Medicine, St George's University, Grenada \\ ${ }^{4}$ Division of Internal Medicine, Center for Healthful Behavior Change, NYU Medical Center, NY 10016, USA \\ ${ }^{5}$ Division of Endocrinology, Department of Medicine, SUNY Downstate Medical Center, NY 11203-2098, USA \\ ${ }^{6}$ Department of Medicine, Sleep Disorders Center, SUNY Downstate Medical Center, NY 11203-2098, USA
}

Correspondence should be addressed to G. Jean-Louis; gjean-louis@downstate.edu

Received 8 October 2012; Revised 28 November 2012; Accepted 6 December 2012

Academic Editor: Juan Carlos Kaski

Copyright (C) 2013 N. Williams et al. This is an open access article distributed under the Creative Commons Attribution License, which permits unrestricted use, distribution, and reproduction in any medium, provided the original work is properly cited.

\begin{abstract}
Background. Evidence shows that blacks exhibit greater daytime sleepiness compared with whites, based on the Epworth Sleepiness Scale. In addition, sleep complaints might differ based on individuals' country of origin. However, it is not clear whether individuals' country of origin has any influence on excessive daytime sleepiness (EDS). Study Objectives. We tested the hypothesis that USborn blacks would show a greater level of EDS compared with foreign-born blacks. The potential effects of sociodemographic and medical risk were also determined. Design. We used the Counseling African-Americans to Control Hypertension (CAATCH) data. CAATCH is a group randomized clinical trial that was conducted among 30 community healthcare centers in New York, yielding baseline data for 1,058 hypertensive black patients. Results. Results of univariate logistic regression analysis indicated that US-born blacks were nearly twice as likely as their foreign-born black counterparts to exhibit EDS (OR = 1.87, 95\% CI: 1.30-2.68, $P<0.001$ ). After adjusting for effects of age, sex, education, employment, body mass index, alcohol consumption, and smoking habit, US-born blacks were $69 \%$ more likely than their counterparts to exhibit EDS (OR $=1.69,95 \%$ CI: 1.11-2.57, $P<0.01)$. Conclusion. Findings demonstrate the importance of considering individuals' country of origin, in addition to their race and ethnicity, when analyzing epidemiologic sleep data.
\end{abstract}

\section{Introduction}

There has been an increased interest in the study of the influence of race/ethnicity on sleep in the last decade. This is fueled in part by epidemiologic evidence showing that individuals of the black race/ethnicity are at increased risk for sleep apnea [1]. An important community-based study involving older black and white participants showed that blacks experienced severe sleep apnea with a relative risk twofold as great (relative risk $=2.13$ ) as that of their counterparts [1]. Ethnic disparities are not noted only among older adults. Indeed, in a casecontrol family study of sleep apnea [2], comparing 225 blacks and 622 whites, ages 2 to 86 years, 31\% of blacks versus $10 \%$ of whites had respiratory disturbance index greater than 10 .
Although blacks are characterized by greater sleep disturbance as observed among patients with sleep apnea, epidemiologic evidence has shown that they are less likely to report sleep problems compared with whites [3]. In a previous paper examining racial/ethnic differences in the rate of sleep complaints (defined as either difficulty initiating sleep, difficulty maintaining sleep, or early morning awakening among older adults), we showed that rates of sleep complaints among white men and women were $41 \%$ and $75 \%$, respectively; among black men and women, they were $14 \%$ and $37 \%$, respectively [4]. There is also evidence that sleep complaints might differ on the basis of individuals' country of origin. Results of our previous study conducted in Brooklyn, NY, USA have shown that the prevalence of sleep complaints among African 
Americans was 71\%; among English-Speaking Caribbeans, the prevalence was 34\%; Haitians, 33\%; Dominicans, $73 \%$; Eastern Europeans, 77\%; and European Americans, 70\% [5].

To date, little is known about the influence of country of origin on excessive daytime sleepiness, an important marker of disturbed sleep. Data from a hospital-based sleep clinic have shown that blacks are characterized by greater daytime sleepiness compared with whites based on the Epworth Sleepiness Scale [6]. Using data from the Counseling AfricanAmericans to Control Hypertension (CAATCH) Trial, we tested the hypothesis that US-born blacks would show a greater level of excessive daytime sleepiness compared with foreign-born blacks. The potential effects of sociodemographic and medical risk were also determined.

\section{Methods}

The Counseling African-Americans to Control Hypertension (CAATCH) Trial is a group randomized clinical trial that was performed among 30 community healthcare centers (CHC). For the purpose of the present analysis, we focused on baseline sociodemographic and clinical data. Details of the CAATCH data acquisition procedures have been published previously [7]. From 2006 to 2009, a total of 1,058 patients (mean age: $46.75 \pm 16.23$ years) were recruited and studied to achieve project aims.

2.1. Procedures. Patients were enrolled if they met the following criteria: self-identification as black or AfricanAmerican, were at least 18 years old, were receiving care at the participating $\mathrm{CHC}$ for at least 6 months period, had a diagnosis of hypertension and uncontrolled blood pressure (BP) at the last office visit ( $\mathrm{BP} \geq 140 / 90)$, and were taking at least 1 antihypertensive medication. In addition, all patients must have had uncontrolled BP (systolic BP $\geq 140 \mathrm{~mm} \mathrm{Hg}$ or diastolic $\mathrm{BP} \geq 90 \mathrm{~mm} \mathrm{Hg}$ ) at the time of the consent visit, as measured by BPTru (VSM Medtech, Model BPM-300), an automated oscillometric validated $\mathrm{BP}$ monitor $[3,8]$. Patients were excluded if they were non-English speaking, had an arm circumference of $>42 \mathrm{~cm}$, participated in other HTN-related trials, used home BP monitoring currently, had cognitive impairment with Mini Mental Status Examination (MMSE) score $<24$ for patients with $>8$ th grade education, or MMSE $<17$ for those with an eighth grade education, or were unwilling or unable to complete screening or baseline assessments, or unwilling or unable to sign informed consent [7].

Trained research assistants interviewed the participants to obtain baseline clinical and sociodemographic data used in the present analysis. Excessive daytime sleepiness was assessed using the Epworth Sleepiness Scale $[6,9]$. Validation study showed that the questionnaire has a sensitivity of 0.94, specificity of 0.79 (based on a clinical cut-off of AHI $>5$ ), positive predictive value of 0.91 , and negative predictive value of 0.86 [10]. Patients' sociodemographic variables including age, gender, income, education, and employment were collected using an instrument developed by the Clinical Directors Network (CDN). All enrolled patients provided informed consent under the supervision of the IRB at New York University Medical Center [7].

2.2. Statistical Analysis. Frequency and measures of central tendency were used to describe the sample. ANOVA was used for group mean comparisons, and Chi-square test was employed to assess differences in categorical variables. To test the hypothesis that UBB participants had a greater likelihood of exhibiting EDS than FBB participants, we utilized multivariate logistic regression modeling. Covariates entered in the model were age, sex, education, income, history of alcohol consumption, smoking habit, and body mass index. Before constructing the model, univariate logistic regressions were performed to assess associations between hypothesized predictors and the dependent variable, EDS; only predictors showing a $P$ value $<0.05$ were entered in the final model [11]. All analyses were performed using SPSS 18.0.

\section{Results}

One thousand and fifty-eight participants provided baseline data for the analysis; 73\% were US-born blacks (UBB), 27\% were foreign-born blacks (FBB). As illustrated in Table 1, there were no significant group differences in terms of age, gender, and body mass index. However, FBB participants were more likely to be employed, but less likely to have received more than a high school education, less likely to report alcohol consumption, and less likely to report a smoking history.

Results of univariate logistic regression analysis indicated that UBB participants were nearly twice as likely as their FBB counterparts to exhibit EDS (OR $=1.87$, 95\% CI: $1.30-$ $2.68, P<0.001)$. Since these two groups differed on the basis of sociodemographic and risk profiles, we performed a multivariate logistic regression analysis adjusting for effects of age, sex, education, body mass index, history of smoking, and alcohol consumption. As indicated in Table 2, UBB participants were $68 \%$ more likely than their FBB counterparts to exhibit EDS. Results of each of the factors entered in the model are indicated in Table 2. Figure 1 illustrates the difference in EDS comparing UBB participants with FBB participants.

\section{Discussion}

Excessive daytime sleepiness is an important marker of insufficient nocturnal sleep, which results from a diagnosable sleep disorder or personal decision to reduce the amount of time spent in bed. There is ample evidence that individuals suffering from disorders such as insomnia, narcolepsy, or sleep apnea experience excessive daytime sleepiness [12]. Epidemiologic evidence demonstrates that the average sleep duration has declined from 8 hours, which was the norm 30 years ago, to 6.5 hours [13]. It is important to note that EDS has a considerable social and economic burden. Evidence shows that EDS leads to 100,000 car accidents in the United States annually $[13,14]$. There is also evidence suggesting that the United States loses 16 billion dollars yearly due to loss 
TABLE 1: Comparison of sociodemographic and health risk data of US-born black versus foreign-born black participating in the CAATCH.

Baseline characteristics based on participants' country of origin

\begin{tabular}{lcc} 
Variable & UBB (73\%) & FBB (27\%) \\
\hline Age (mean \pm SD) & $56 \pm 13$ & $58 \pm 13$ \\
Female gender (\%) & 71 & 73 \\
Employed (\%) & 29 & $45^{*}$ \\
High school (\%) & 31 & $23^{*}$ \\
Alcohol consumption (\%) & 39 & $18^{*}$ \\
Smoking history (\%) & 63 & $24^{*}$ \\
Body mass index (mean \pm SD) & $31 \pm 5.74$ & $34 \pm 8.01$ \\
\hline
\end{tabular}

${ }^{*} P<0.001$. Mean $\pm \mathrm{SD}$ and (\%) is reported.

TABLE 2: Regression coefficients of the EDS measure on country of origin (US-Born Black versus Foreign-Born Black), sociodemographic, and risk factors.

Associations of country of origin, sociodemographic, and risk factors with excessive daytime sleepiness

\begin{tabular}{lccc} 
Variable & OR & $95 \%$ CI & $P$ \\
\hline US-Born Black & 1.679 & $1.103-2.555$ & 0.02 \\
Age & 0.989 & $0.975-1.004$ & 0.15 \\
Sex & 0.857 & $0.598-1.227$ & 0.40 \\
Education & 0.908 & $0.786-1.049$ & 0.19 \\
Employed & 1.038 & $0.718-1.501$ & 0.84 \\
Alcohol consumption & 0.850 & $0.588-1.227$ & 0.39 \\
Smoking habit & 1.033 & $0.723-1.477$ & 0.86 \\
Body mass index & 1.000 & $1.000-1.000$ & 0.53 \\
\hline
\end{tabular}

of productivity, hours spent away from work, and medical conditions related to daytime sleepiness [15]. In light of these findings, public health advocates have been concerned that certain vulnerable populations might be at disproportionately worse levels of EDS-associated risk [16].

The main finding of our study is that UBB participants were nearly twice as likely as their FBB counterparts to exhibit EDS. This association remained significant even after adjusting for effects of sociodemographic and medical risk factors. To our knowledge, this is the first study that shows differences in EDS based on individuals' country of origin. Of note, levels of EDS observed for both groups were comparable to levels of EDS found in the Multiethnic Study of Atherosclerosis [17]. These data seem consistent with a previous community-based study we conducted [4]. In sum, these findings demonstrate further the importance of considering country of origin in the analysis of the epidemiologic sleep data. Thus, previous epidemiologic studies aggregating individuals from different countries of origin into one stratum (e.g., black, Hispanic, white, and others) may have been biased.

There are no data suggesting greater prevalence of sleep apnea among UBB compared with FBB. Since EDS is one of the main symptoms of sleep apnea, and because these patients have an existing diagnosis of hypertension, UBB may be at greater risk for sleep apnea, relative to their FBB counterparts.

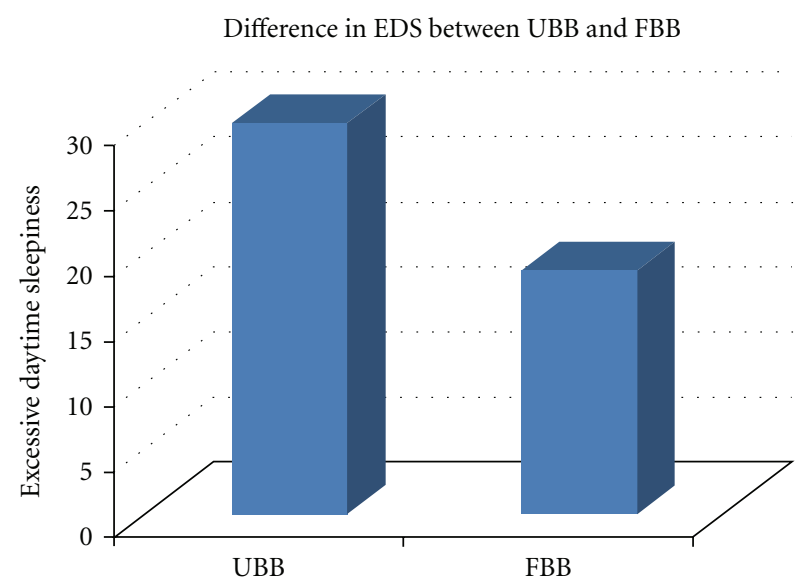

FIGURE 1: Illustration of difference in excessive daytime sleepiness between US-born blacks and foreign-born blacks $\left(\chi^{2}=12.61, P<\right.$ $0.001)$.

Unfortunately, the presence of and severity of sleep apnea was not assessed in this study. Hence, it is not clear why UBB have greater prevalence of EDS. If in fact it can be demonstrated that indeed UBB have greater prevalence of sleep apnea, public health efforts should be made to highlight the need for more research to understand why UBB are at greater risk for this condition. These findings also point to the need to examine the interrelationship among genetic [18], environmental $[19,20]$, and lifestyle [21] factors that might predispose one group to develop sleep apnea, more so than others.

Despite these findings there are important limitations to our study that should be noted. Our analyses were performed using subjective data, which do not always correspond well with objective data. Future studies should determine whether FBB and UBB will show differences in EDS measured objectively. Another important limitation of our study is that we did not have a control group. It would be important to assess the difference in the prevalence of EDS comparing hypertensive blacks with normotensive blacks.

\section{Conclusions}

Results of our study are important regarding evidence suggesting that individuals' country of origin should be considered in the analysis of epidemiologic sleep data in addition to their race and ethnicity. Specifically, we found that USborn hypertensive blacks are more likely to experience EDS compared with foreign-born hypertensive blacks.

\section{Conflict of Interests}

The authors declare no conflict of interests.

\section{Acknowledgment}

This research was supported by funding from the NIH (R25HL105444, R01HL095799, R01MD004113, R01HL078566, and K24HL111315). 


\section{References}

[1] S. Ancoli-Israel, M. R. Klauber, C. Stepnowsky, E. Estline, A. Chinn, and R. Fell, "Sleep-disordered breathing in AfricanAmerican elderly," American Journal of Respiratory and Critical Care Medicine, vol. 152, no. 6, pp. 1946-1949, 1995.

[2] B. El-Ad and P. Lavie, "Effect of sleep apnea on cognition and mood," International Review of Psychiatry, vol. 17, no. 4, pp. 277282, 2005.

[3] C. Allison, "BpTRU(tm) blood pressure monitor for use in a physician's office," Issues in Emerging Health Technologies, no. 86, pp. 1-4, 2006.

[4] G. Jean-Louis, C. M. Magai, C. I. Cohen et al., "Ethnic differences in self-reported sleep problems in older adults," Sleep, vol. 24, no. 8, pp. 926-933, 2001.

[5] G. Jean-Louis, C. Magai, G. J. Casimir et al., "Insomnia symptoms in a multiethnic sample of American women," Journal of Women's Health, vol. 17, no. 1, pp. 15-25, 2008.

[6] A. L. Hayes, J. C. Spilsbury, and S. R. Patel, "The Epworth score in African American populations," Journal of Clinical Sleep Medicine, vol. 5, no. 4, pp. 344-348, 2009.

[7] G. Ogedegbe, J. N. Tobin, S. Fernandez et al., "Counseling African Americans to control hypertension (CAATCH) trial: a multi-level intervention to improve blood pressure control in hypertensive blacks," Circulation: Cardiovascular Quality and Outcomes, vol. 2, no. 3, pp. 249-256, 2009.

[8] M. G. Myers, M. Valdivieso, and A. Kiss, "Use of automated office blood pressure measurement to reduce the white coat response," Journal of Hypertension, vol. 27, no. 2, pp. 280-286, 2009.

[9] M. W. Johns, "A new method for measuring daytime sleepiness: the Epworth sleepiness scale," Sleep, vol. 14, no. 6, pp. 540-545, 1991.

[10] D. J. Levendowski, E. M. Olmstead, D. Popovich, D. Carper, C. Berka, and P. R. Westbrook, "Assessment of obstructive sleep apnea risk and severity in truck drivers: validation of a screening questionnaire," Sleep Diagnosis and Therapy, vol. 2, pp. 20-26, 2007.

[11] D. W. Hosmer, S. Taber, and S. Lemeshow, "The importance of assessing the fit of logistic regression models: a case study," American Journal of Public Health, vol. 81, no. 12, pp. 1630-1635, 1991.

[12] J. F. Pagel, "Excessive daytime sleepiness," American Family Physician, vol. 79, no. 5, pp. 391-396, 2009.

[13] G. Jean-Louis, D. F. Kripke, S. Ancoli-Israel, M. R. Klauber, and R. S. Sepulveda, "Sleep duration, illumination, and activity patterns in a population sample: effects of gender and ethnicity," Biological Psychiatry, vol. 47, no. 10, pp. 921-927, 2000.

[14] D. Drobnich, “A national sleep foundation's conference summary: the national summit to prevent drowsy driving and a new call to action," Industrial Health, vol. 43, no. 1, pp. 197-200, 2005.

[15] D. Rodenstein, "Sleep apnea: traffic and occupational accidents-individual risks, socioeconomic and legal implications," Respiration, vol. 78, no. 3, pp. 241-248, 2009.

[16] C. M. Baldwin, A. M. Ervin, M. Z. Mays et al., "Sleep disturbances, quality of life, and ethnicity: the Sleep Heart Health Study," Journal of Clinical Sleep Medicine, vol. 6, no. 2, pp. 176$183,2010$.

[17] K. G. Baron, K. Liu, C. Chan, E. Shahar, R. Hasnain-Wynia, and P. Zee, "Race and ethnic variation in excessive daytime sleepiness: the multi-ethnic study of atherosclerosis," Behavioral Sleep Medicine, vol. 8, no. 4, pp. 231-245, 2010.
[18] B. D. Kent, S. Ryan, and W. T. McNicholas, "The genetics of obstructive sleep apnoea," Current Opinion in Pulmonary Medicine, vol. 16, no. 6, pp. 536-542, 2010.

[19] M. J. Doherty, C. E. Youn, A. M. Haitiner, and N. F. Watson, "Do weather-related ambient atmospheric-pressure changes influence sleep disordered breathing?” Journal of Clinical Sleep Medicine, vol. 6, no. 2, pp. 152-156, 2010.

[20] Y. Nussbaumer-Ochsner, N. Schuepfer, S. Ulrich, and K. E. Bloch, "Exacerbation of sleep apnoea by frequent central events in patients with the obstructive sleep apnoea syndrome at altitude: a randomised trial," Thorax, vol. 65, no. 5, pp. 429-435, 2010.

[21] H. G. Lund, B. D. Reider, A. B. Whiting, and J. R. Prichard, "Sleep patterns and predictors of disturbed sleep in a large population of college students," Journal of Adolescent Health, vol. 46, no. 2, pp. 124-132, 2010. 


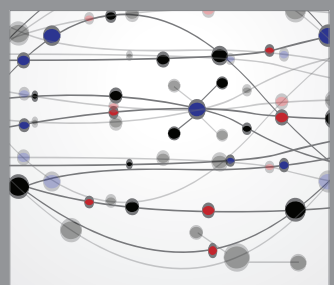

The Scientific World Journal
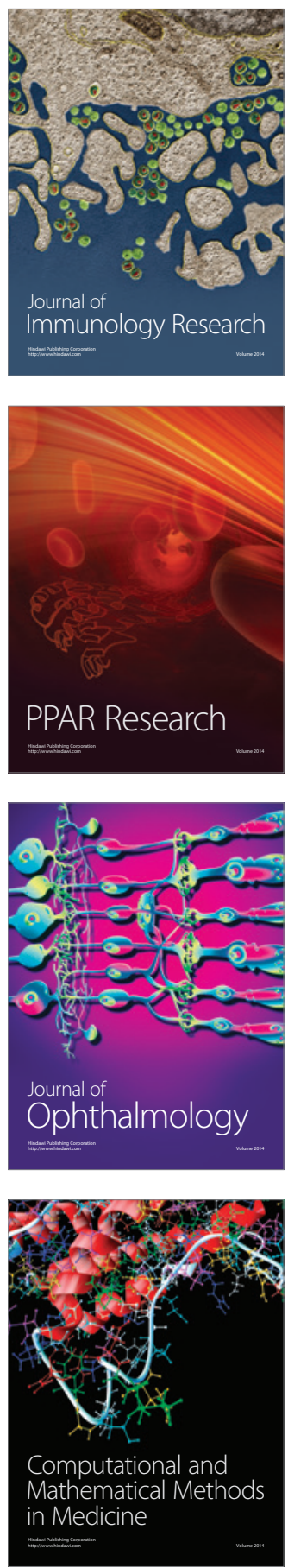

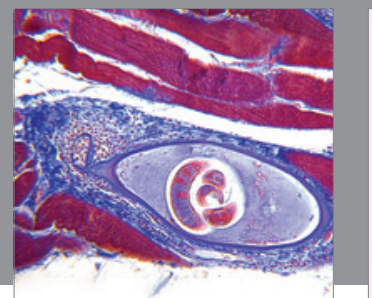

Gastroenterology

Research and Practice
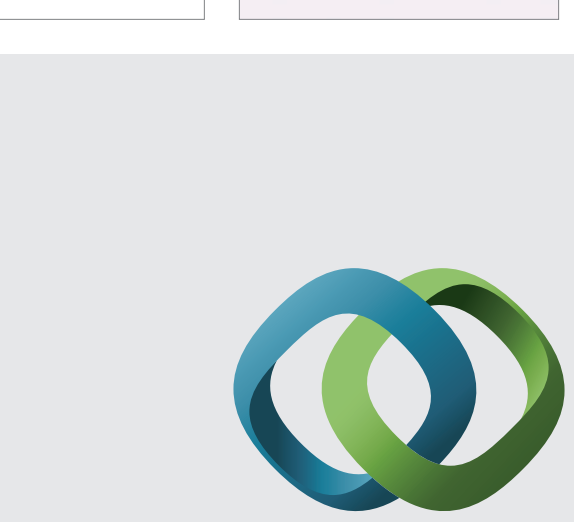

\section{Hindawi}

Submit your manuscripts at

http://www.hindawi.com
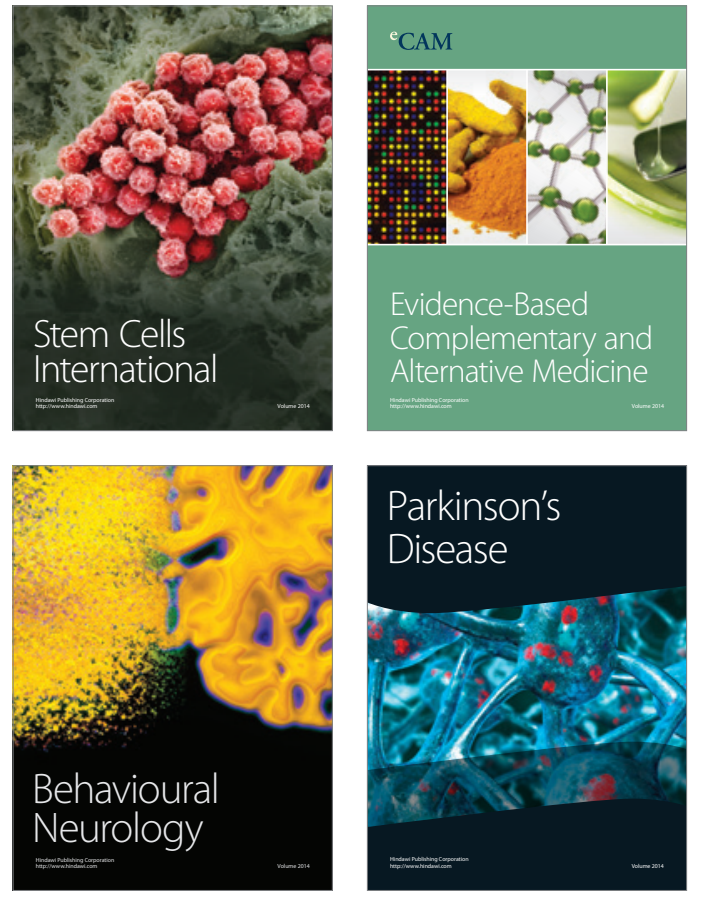
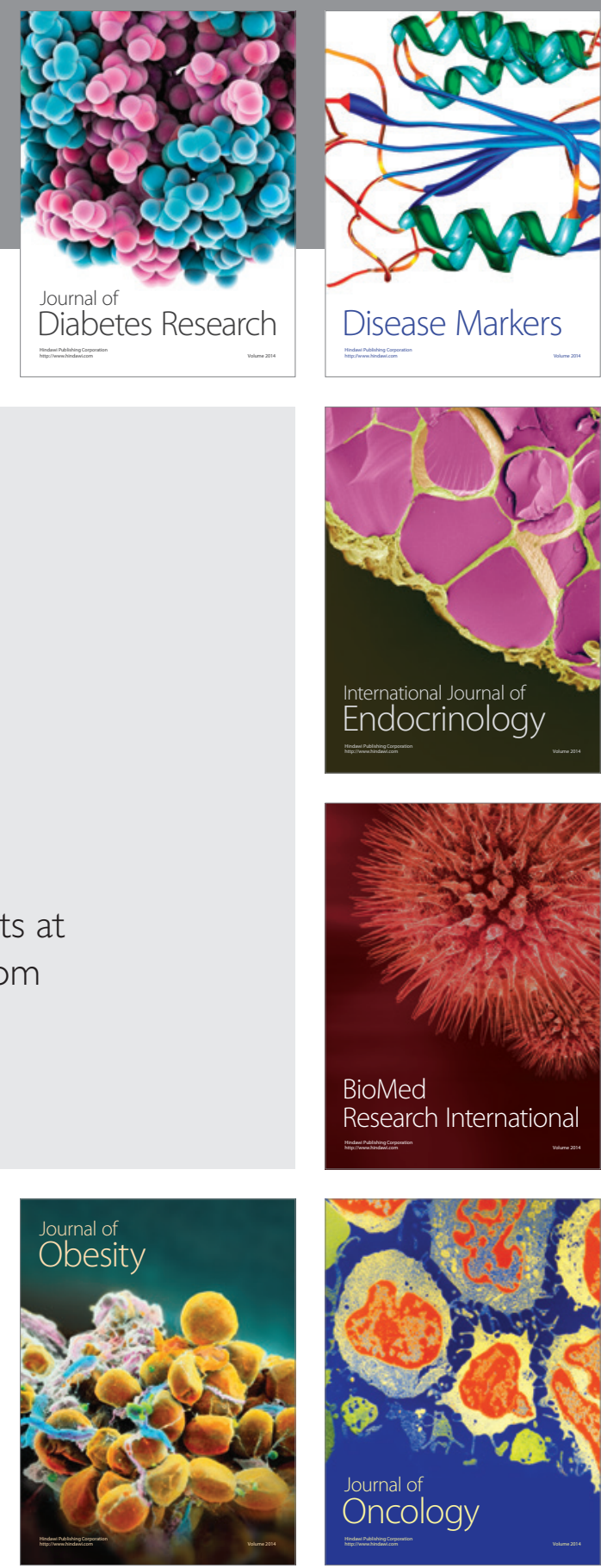

Disease Markers
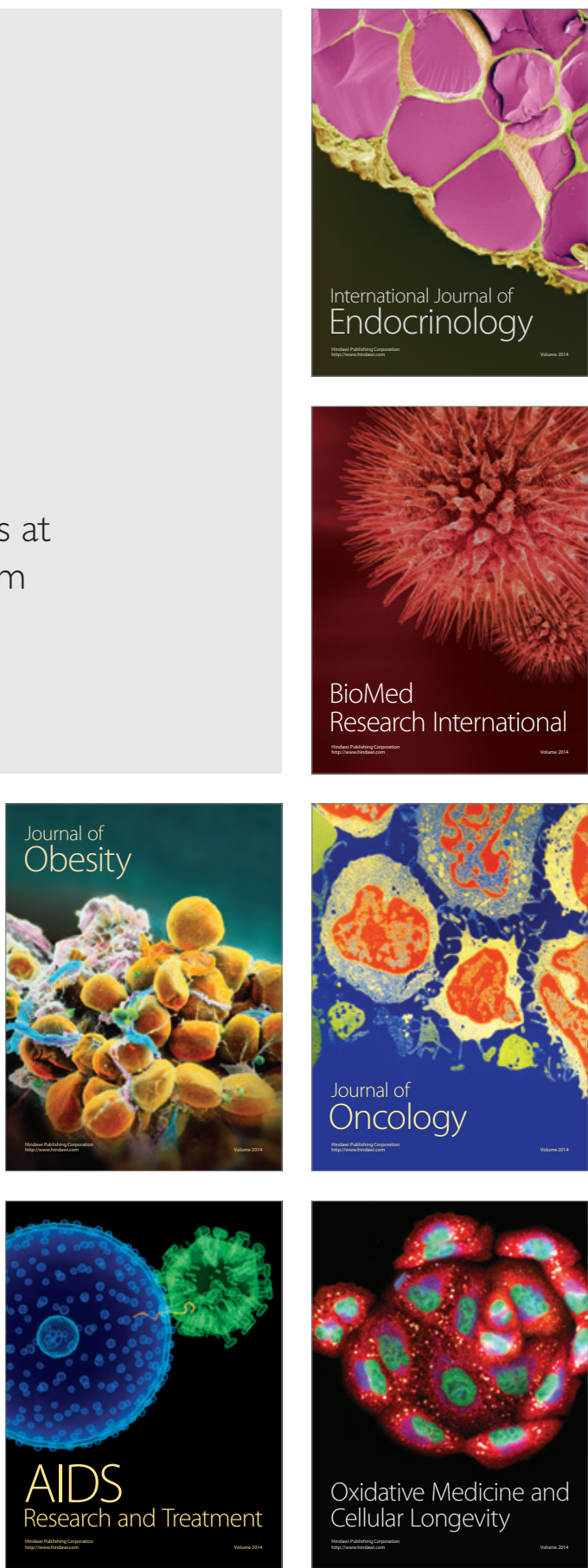